\title{
Safety and Efficacy of the Sirolimus Gel for TSC Patients With Facial Skin Lesions in a Long-Term, Open-Label, Extension, Uncontrolled Clinical Trial
}

\author{
Mari Wataya-Kaneda · Hiroshi Nagai · Yuuki Ohno · Hiroo Yokozeki · Yasuyuki Fujita · Hironori Niizeki • \\ Kazue Yoshida · Masaaki Ogai • Yuichi Yoshida • Akihiko Asahina • Kazuyoshi Fukai • Chiharu Tateishi • \\ Izumi Hamada $\cdot$ Tatsuro Takahata $\cdot$ Kenji Shimizu $\cdot$ Shigeki Shimasaki $\cdot$ Hiroyuki Murota
}

Received: March 30, 2020 / Published online: May 8, 2020

(C) The Author(s) 2020

\section{ABSTRACT}

Introduction: Our previous clinical studies have demonstrated the short-term efficacy and safety of the sirolimus gel for patients with tuberous sclerosis complex (TSC). However, long-term clinical evidence is lacking. Our objective was to assess the safety and efficacy of long-term treatment with the sirolimus gel for the skin lesions of TSC patients.

Digital Features To view digital features for this article go to https://doi.org/10.6084/m9.figshare.12162036.

M. Wataya-Kaneda $(\bowtie)$

Department of Dermatology, Graduate School of

Medicine, Osaka University, Osaka, Japan

e-mail: mkaneda@derma.med.osaka-u.ac.jp

\section{H. Nagai}

Division of Dermatology, Department of Internal

Related, Kobe University Graduate School of

Medicine, Hyogo, Japan

Y. Ohno

Department of Dermatology, JR Tokyo General

Hospital, Tokyo, Japan

H. Yokozeki

Department of Dermatology, Medical Hospital,

Tokyo Medical and Dental University, Tokyo, Japan

Y. Fujita

Department of Dermatology, Hokkaido University

Graduate School of Medicine, Hokkaido, Japan
Methods: We conducted a multicenter, openlabel, uncontrolled clinical trial in 94 Japanese patients with TSC. Patients applied the $0.2 \%$ sirolimus gel on their face or head twice daily for $>$ 52 weeks (maximum 136 weeks for safety). The safety endpoints were the rate of adverse event (AE)-caused discontinuation (primary endpoint) and the incidence of AEs. The efficacy endpoint was the response rate of angiofibromas, cephalic plaques, and hypomelanotic macules.

Results: Among 94 enrolled patients (mean age, 21 years; range 3-53 years), the rate of AEcaused discontinuation was $2.1 \% \quad(2 / 94$ patients). Although application site irritation and dry skin occurred relatively frequently,

H. Niizeki · K. Yoshida

Division of Dermatology, Department of Surgical Subspecialties, National Center for Child Health and Development, Tokyo, Japan

M. Ogai

Department of Dermatology, Seirei Hamamatsu

General Hospital, Shizuoka, Japan

Y. Yoshida

Division of Dermatology, Department of Medicine of Sensory and Motor Organs, Faculty of Medicine, Tottori University, Tottori, Japan

\section{A. Asahina}

Department of Dermatology, The Jikei University

School of Medicine, Tokyo, Japan

K. Fukai · C. Tateishi

Department of Dermatology, Osaka City University

Graduate School of Medicine, Osaka, Japan 
none of the drug-related AEs were serious; most of the drug-related AEs resolved rapidly. The major drug-related AEs ( $\geq 5 \%$ in incidence) were application site irritation (30.9\%), dry skin $(27.7 \%)$, acne $(20.2 \%)$, eye irritation $(8.5 \%)$, pruritus $(8.5 \%)$, erythema $(7.4 \%)$, dermatitis acneiform (6.4\%), and dermatitis contact $(5.3 \%)$. The response rates of angiofibromas, cephalic plaques, and hypomelanotic macules were $78.2 \%[95 \%$ confidence interval (CI) 68.0-86.3\%], 66.7\% (95\% CI 51.1-80.0\%), and 72.2\% (95\% CI 46.5-90.3\%), respectively.

Conclusions: The gel was well tolerated for a long time by patients with TSC involving facial skin lesions and continued to be effective.

Trial Registration: ClinicalTrials.gov identifier: NCT02634931.

Keywords: Angiofibromas; Cephalic plaques; Clinical trial; Hypomelanotic macules; Longterm administration; Sirolimus gel; Skin lesions; Topical sirolimus; Tuberous sclerosis complex

\section{Key Summary Points}

\section{Why carry out this study?}

Tuberous sclerosis complex (TSC), an autosomal dominant genetic disorder with an incidence rate of approximately 1 in 5000-10,000 live births, causes hamartomas in various organs including the skin.

We previously verified the clinical usefulness of the $0.2 \%$ sirolimus gel in a 12 -week, phase 3 clinical trial of patients with TSC involving angiofibromas. However, the long-term tolerability and efficacy of the gel are not sufficiently documented.

\section{K. Fukai}

Department of Dermatology, Osaka City General Hospital, Osaka, Japan

I. Hamada · T. Takahata - K. Shimizu · S. Shimasaki Nobelpharma Co., Ltd, Tokyo, Japan

H. Murota

Department of Dermatology, Nagasaki University, Nagasaki, Japan
To confirm the safety and efficacy of longterm treatment with the sirolimus gel for TSC skin lesions, we conducted a multicenter, open-label, uncontrolled clinical trial of TSC patients at 10 sites in Japan.

\section{What was learned from the study?}

The response rate of TSC patients to gel treatment continued to increase for the 52-week evaluation period, and the gel improved the size and color of severe facial angiofibromas as well.

The sirolimus gel, when applied topically for a long period of time, was well tolerated by and effective for TSC patients including those with severe facial skin lesions.

\section{INTRODUCTION}

Tuberous sclerosis complex (TSC), an autosomal dominant genetic disorder caused by TSC1 or TSC2 mutations, is characterized by hamartomas in various organs (e.g., skin, brain, and kidneys). TSC provokes skin lesions (e.g., hypomelanotic macules, angiofibromas, and cephalic plaques), epilepsy, neurodevelopmental disorders, and other clinical manifestations [1-3]. Regarding skin lesions, hypomelanotic macules develop at birth or later in the majority of affected newborns. Facial angiofibromas usually appear by age 5 and gradually proliferate thereafter. Facial skin lesions deteriorate as TSC progresses on a yearly basis, which adversely affects the quality of life of patients by causing psychologic and social distress [4-7].

Oral mammalian target of rapamycin complex 1 (mTORC1) inhibitors are effective for subependymal giant cell astrocytoma, renal angiomyolipoma, lymphangioleiomyomatosis (LAM), epilepsy associated with TSC, and skin lesions [8-11]. These oral mTORC1 inhibitors are commercialized in many countries. Efforts have been made to formulate topical mTORC1 inhibitors, and several studies on these drugs have been published [12-17]. We previously 
verified the clinical usefulness of $0.2 \%$ sirolimus gel in a 12-week, phase 3 clinical trial of patients with TSC involving angiofibromas [15]. Patients with TSC need lifelong treatment with the gel because its discontinuation causes the recurrence of angiofibromas. However, clinical evidence on the long-term efficacy and safety of the treatment is lacking. In this long-term extension study lasting for $\geq 52$ weeks, we used the gel to treat Japanese TSC patients with facial skin lesions for assessing its long-term safety and efficacy.

\section{METHODS}

\section{Study Design and Patients}

This was a long-term, open-label, extension, uncontrolled clinical trial designed to enroll a part of patients who had been enrolled in our previous phase 3 randomized clinical trial of the sirolimus gel [15] and to newly enroll patients. Patients were eligible if they were aged $\geq$ 3 years, met the definite diagnosis of TSC [18], and had angiofibromas, cephalic plaques, or hypomelanotic macules on the face or head. The key exclusion criteria were as follows: (1) a patient who withdrew from the prior phase 3 trial of the gel; (2) a patient who had skin inflammation manifested by erosion, ulcer, and eruption at the application site; (3) a patient who had comorbidities (e.g., serious disorders and poorly controlled diabetes mellitus and dyslipidemia). The present open-label, uncontrolled clinical trial was conducted at ten medical institutions in Japan. The present study was conducted between February 2016 and September 2018. This study was conducted in accordance with the principles of the Declaration of Helsinki of 1964 and its later amendments and in compliance with clinical practices and local regulatory requirements. This study was approved by the institutional review board (IRB) at each institution (see acknowledgements for full IRB list). Prior to enrollment, written informed consent was obtained from competent adult patients and from the legal representatives of patients $<20$ years of age or patients with intellectual impairment. Patients also provided consent for publication.

\section{Procedures}

The $0.2 \%$ sirolimus gel, provided by Nobelpharma Co., Ltd., Tokyo, Japan, contained sirolimus as active ingredient as well as ethanol and carboxyvinyl polymer as additives. The gel had to be applied twice daily (morning and before bedtime) at an approximate dose of $125 \mathrm{mg}$ per $50 \mathrm{~cm}^{2}$ of application site on a finger-tip unit basis. The upper limit for daily dose was set to be $400 \mathrm{mg}, 600 \mathrm{mg}$, and $800 \mathrm{mg}$ for children aged $<6$ years, 6-11 years, and $\geq 12$ years, respectively. Topical application was continued unless falling under any one of the exclusion criteria and as long as the patient desired treatment. However, treatment had to last until the date of availability of the commercialized gel at the medical institution after regulatory approval or up to September 2018, whichever was earlier. Patients visited the hospital at screening, at baseline, as well as at weeks $4,8,12,16,21,26,39$, and 52 of treatment, and every 3 months thereafter. The efficacy of the gel was assessed for the first 52 weeks of treatment. Skin lesions on the patient's face were photographed with a digital camera (Olympus STYLUS TG-4 Tough, Tokyo, Japan) that was used in common at all medical institutions. A color chart with the scale to adjust the tones and clarity of color images and lesion size, CASMATCH ${ }^{\mathrm{TM}}$ (Bear Medic, Ibaraki, Japan), was used at the time of photography. After a hospital visit at week 52 of treatment, patients were assessed for safety alone. The concurrent use of medications other than the gel (e.g., topical/oral steroids, topical/oral antibiotics, moisturizers, oral mTORC1 inhibitors) was allowed during the trial. Whole blood was collected at an arbitrary time during the visit to measure blood sirolimus concentrations by liquid chromatography-tandem mass spectrometry. 


\section{Assessments}

The primary endpoint was the rate of AE-caused discontinuation. At each hospital visit, the safety of the gel was assessed through history taking, physical examination, laboratory tests (hematology, blood chemistry, and urinalysis), as well as vital signs. In addition to hospital visits, patients were contacted by phone to collect information on any AEs. Blood sirolimus concentrations were measured at each hospital visit for the first 52 weeks of treatment.

The efficacy endpoints were a composite improvement in angiofibromas-combined improvements in size and color-as well as in cephalic plaques and hypomelanotic macules, and changes in DLQI/CDLQI [19, 20], and patients' satisfaction with treatment outcomes was assessed based on the patient- or surrogate consenter-reported questionnaire $(1$, very satisfied; 2 , satisfied; 3 , undeterminable; 4 , unsatisfied; 5 , very unsatisfied); these endpoints were assessed from the onset of application to week 52 of treatment. The method of efficacy assessment was identical to that used for the phase 3 randomized clinical trial [15]. Namely, the independent review committee (IRC) consisting of three dermatologists assessed the photographs of patients that had been taken at weeks $4,8,12,26,39$, and 52 of treatment to compare them with those taken at baseline according to the following six-category criterion: markedly improved, improved, slightly improved, unchanged, slightly aggravated, and aggravated. Additionally, the proportion of patients who were adjudicated by the IRC to "markedly improved" or "improved" was considered as the improvement rate.

\section{Statistical Methods}

Sample size was not calculated statistically but was established to be at least 80 patients based on the maximum expected number of patients who were enrollable at medical institutions in our previous phase 3 randomized clinical trial [15]. Patients with TSC, who had been enrolled in the previous phase 3 clinical trial of the gel, were also considered enrollable in the present trial. The preferred terms of the medical dictionary for regulatory activities (MedDRA version 20.1) were used for reporting AEs. The cumulative incidence of drug-related AEs ( $\geq 5 \%$ in incidence) was calculated according to the cumulative incidence function of Kaplan-Meier estimates. Fisher's exact test was conducted to compare the response rates between two subgroups. Blood sirolimus concentrations at each visit were analyzed using linear regression models with baseline value as a covariate and background factors as the fixed effect. A value of $P<0.05$ was considered statistically significant. The SAS software package version 9.4 (SAS Inc., Cary, NC, USA) was used for statistical analyses and the R package version 3.4.1 for drawing the cumulative incidences and duration of drug-related AEs estimated by the Kaplan-Meier method.

\section{RESULTS}

Ninety-five patients were screened. After exclusion of one ineligible patient, 94 patients were enrolled in the present clinical trial (Fig. 1), namely, 62 who had completed treatment in our previous phase 3 randomized clinical trial of the sirolimus gel [15] and 32 new ones. The gel was applied to 94 patients with TSC (44 adults and 50 children) for a maximum of 136 weeks. The demographic and clinical characteristics of patients are shown in Table 1 . The gel was applied to 88 patients for $\geq 52$ weeks, 53 of whom continued the application for $\geq 104$ more weeks. The application was discontinued because of drug-related AEs only in two patients (2.1\%): one due to eye irritation and erythema on day 15 of treatment and another due to dermatitis contact on day 29 of treatment. Both AEs were mild in severity and required no treatment. These AEs resolved rapidly after discontinuation, and other reasons for discontinuation are shown in Fig. 1.

The incidences of drug-related AEs were $76.6 \%, 72.7 \%$, and $80.0 \%$ for the whole, adults, and children, respectively. The major drug-related AEs ( $\geq 5 \%$ in incidence) were application site irritation $(30.9 \%)$, dry skin $(27.7 \%)$, acne $(20.2 \%)$, eye irritation (8.5\%), pruritus $(8.5 \%)$, 


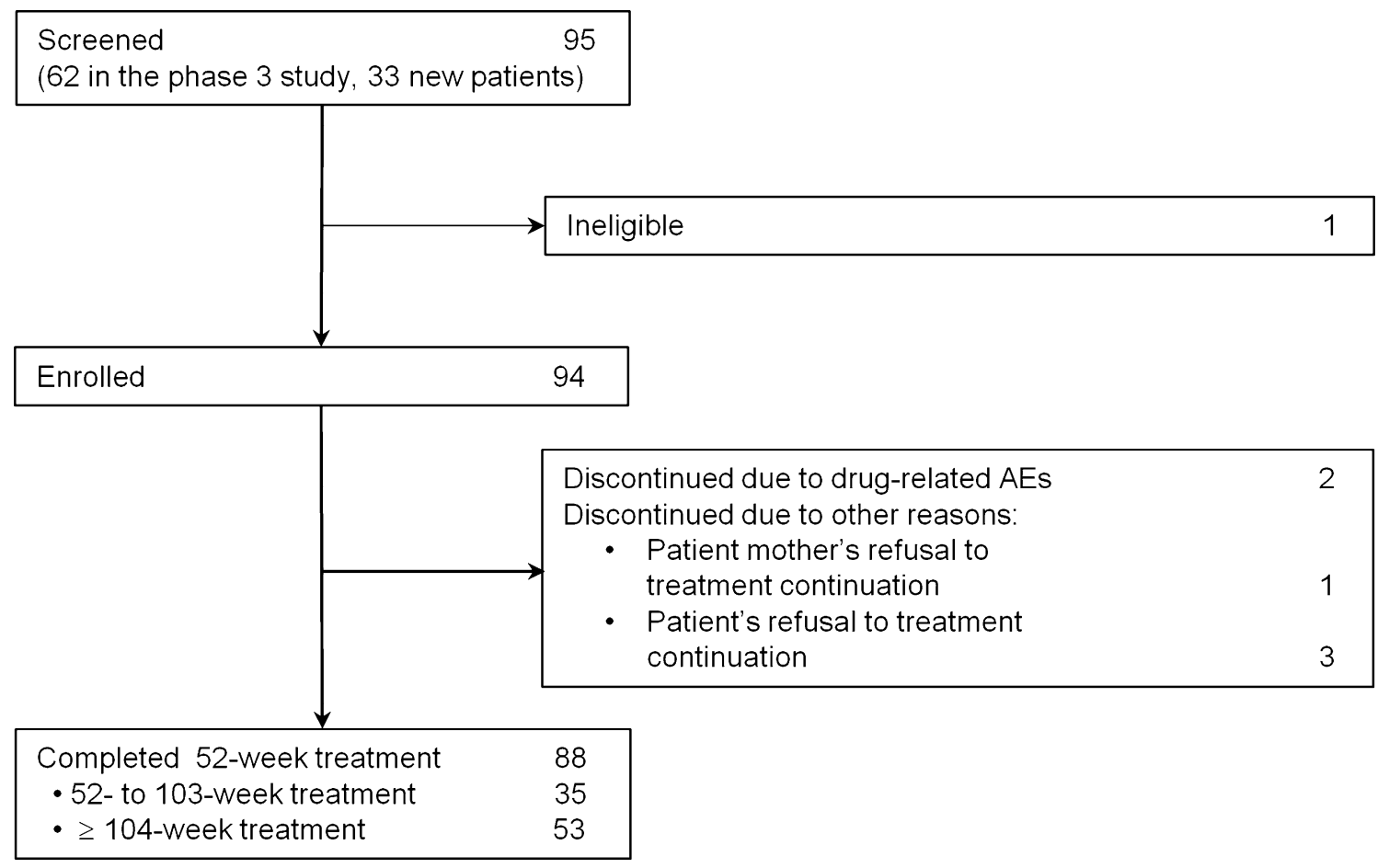

Fig. 1 Patient disposition. Ninety-four patients with TSC were enrolled in the present clinical trial, 62 of whom had been enrolled in the 12 -week phase 3 clinical trial (the sirolimus $0.2 \%$ gel group: 30 patients; the placebo group: 32 patients). Thirty-three patients were newly enrolled. The gel treatment was discontinued in six patients because of AEs or other reasons prior to week 52 of treatment, and

erythema (7.4\%), dermatitis acneiform (6.4\%), and dermatitis contact (5.3\%). Figure 2 shows the cumulative incidences of these major drugrelated AEs, among which application site irritation and dry skin increased markedly up to week 26 of treatment and less pronouncedly thereafter. Acneiform dermatitis increased over time. Furthermore, the incidences of all these drug-related AEs reduced to half or below at week 68 from their onset (Fig. 3). Regarding AEs of clinical concern, solar dermatitis and photosensitivity were found in two patients $(2.1 \%)$. The former was treated with tacrolimus ointment and hydrocortisone butyrate ester cream and by the 10-day holiday from the gel treatment. The latter was treated with the olopatadine hydrochloride tablet, hydrocortisone butyrate ester ointment, and diflupredonate ointment, without requiring any holiday from
88 patients continued the treatment thereafter. All of the latter were considered to have completed the treatment. The present clinical trial was terminated when the gel became commercially available in Japan. This cohort of patients includes patients for whom the treatment was discontinued for other reasons at week 52 of treatment or later. TSC tuberous sclerosis complex, $A E s$ adverse events

the gel treatment. Nine cases $(9.6 \%)$ of serious AEs (e.g., corpus callosotomy, pharyngitis, pneumothorax, wisdom tooth removal, and therapeutic embolism) were found. However, the causality of all these serious AEs with the gel was denied. Throughout the study period, laboratory values and vital signs did not show any abnormal changes of clinical concern.

The response rates of angiofibromas for the whole increased rapidly during the first 12 weeks of treatment and increased gradually thereafter to become $78.2 \% \quad(95 \% \quad \mathrm{CI}$ $68.0-86.3 \%$ ) at week 52 of treatment (Fig. 4a). In children, the improvement rates of angiofibromas reached $74.5 \%$ (95\% CI 59.7-86.1\%) at week 12 of treatment and remained nearly at a plateau up to week 52 of treatment. In adults, the improvement rates of angiofibromas increased linearly to become $41.5 \%$ (95\% CI 
Table 1 Demographic and clinical characteristics of patients as well as concurrent medications and durations of treatment

\begin{tabular}{|c|c|c|c|}
\hline Characteristics & Categories & $\begin{array}{l}\text { Adults } \\
(n=44)\end{array}$ & $\begin{array}{l}\text { Children } \\
(n=50)\end{array}$ \\
\hline \multirow[t]{2}{*}{ Sex, $n(\%)$} & Male & $17(38.6)$ & $24(48.0)$ \\
\hline & Female & $27(61.4)$ & $26(52.0)$ \\
\hline \multirow[t]{5}{*}{ Age, $n(\%)$} & $3-5$ years & - & $4(8.0)$ \\
\hline & $6-11$ years & - & $22(44.0)$ \\
\hline & $12-18$ years & - & $24(48.8)$ \\
\hline & $\geq 19$ years & $44(100)$ & - \\
\hline & Mean & $31.7(10.0)$ & $11.7(4.0)$ \\
\hline Height $(\mathrm{cm})$ & Mean (SD) & $161.9(7.9)$ & $143.0(19.6)$ \\
\hline Body weight (kg) & Mean (SD) & $58.1(12.8)$ & $38.3(14.7)$ \\
\hline \multirow[t]{11}{*}{$\begin{array}{l}\text { TSC major manifestations, } \\
n(\%)\end{array}$} & $\begin{array}{l}\text { Hypomelanotic macules ( } \geq 3 \text {, at least } 5 \mathrm{~mm} \text { in } \\
\text { diameter) }\end{array}$ & $25(56.8)$ & $36(72.0)$ \\
\hline & Angiofibromas $(\geq 3)$ or cephalic plaques & $44(100)$ & $50(100)$ \\
\hline & Ungual fibromas $(\geq 2)$ & $26(59.1)$ & $6(12.0)$ \\
\hline & Shagreen patch & $28(63.6)$ & $29(58.0)$ \\
\hline & Multiple retinal hamartomas & $8(18.2)$ & $20(40.0)$ \\
\hline & Cortical dysplasias & $26(59.1)$ & $31(62.0)$ \\
\hline & Subependymal nodules & $27(61.4)$ & $41(82.0)$ \\
\hline & Subependymal giant cell astrocytomas & $4(9.1)$ & $6(12.0)$ \\
\hline & Cardiac rhabdomyoma & $3(6.8)$ & $16(32.0)$ \\
\hline & Lymphangioleiomyomatosis & $15(34.1)$ & $1(2.0)$ \\
\hline & Angiomyolipomas $(\geq 2)$ & $32(72.7)$ & $24(48.0)$ \\
\hline \multirow{6}{*}{$\begin{array}{l}\text { TSC minor manifestations, } \\
n(\%)\end{array}$} & Confetti skin lesions & $3(6.8)$ & $3(6.0)$ \\
\hline & Dental enamel pits $(\geq 3)$ & $12(27.3)$ & $11(22.0)$ \\
\hline & Intraoral fibromas $(\geq 2)$ & $9(20.5)$ & $4(8.0)$ \\
\hline & Retinal achromic patch & $3(6.8)$ & $0(0.0)$ \\
\hline & Multiple renal cysts & $9(20.5)$ & $9(18.0)$ \\
\hline & Nonrenal hamartomas & $11(25.0)$ & $3(6.0)$ \\
\hline \multirow[t]{3}{*}{ Complications, $n(\%)$} & Epilepsy & $21(47.7)$ & $39(78.0)$ \\
\hline & Neurodevelopmental disorders* & $15(34.1)$ & $30(60.0)$ \\
\hline & Seasonal allergy & $9(20.5)$ & $6(12.0)$ \\
\hline
\end{tabular}


Table 1 continued

\begin{tabular}{|c|c|c|c|}
\hline Characteristics & Categories & $\begin{array}{l}\text { Adults } \\
(n=44)\end{array}$ & $\begin{array}{l}\text { Children } \\
(n=50)\end{array}$ \\
\hline \multirow{7}{*}{$\begin{array}{l}\text { Concurrent medications, } \\
n(\%)\end{array}$} & Oral mTORC1 inhibitors & $9(20.5)$ & $10(20.0)$ \\
\hline & Heparinoid (Hirudoid ${ }^{\circledR}$ ) & $18(40.9)$ & $20(40.0)$ \\
\hline & Petrolatum & $16(36.4)$ & $20(40.0)$ \\
\hline & NSAIDs & $14(31.8)$ & $10(20.0)$ \\
\hline & Antiepileptic drugs & $22(50.0)$ & $36(72.0)$ \\
\hline & Antibiotics (topical) & $27(61.4)$ & $30(60.0)$ \\
\hline & Steroids (topical) & $20(45.5)$ & $32(64.0)$ \\
\hline \multirow[t]{3}{*}{ Durations of treatment, $n(\%)$} & $<52$ weeks & $4(9.1)$ & $1(2.0)$ \\
\hline & $52-103$ weeks & $10(22.7)$ & $10(20.0)$ \\
\hline & $\geq 104$ weeks & $30(68.2)$ & $39(78.0)$ \\
\hline
\end{tabular}

mTORC1 mammalian target of rapamycin complex 1, NSAIDs nonsteroidal anti-inflammatory drugs

"Include "intellectual disability," "trisomy 21 ," and "autism spectrum disorder"

26.3-57.9\%) and $82.1 \%(95 \%$ CI 66.5-92.5\%) at weeks 12 and 52 of treatment, respectively; a statistically significant difference was found between adults and children at week 12 of treatment but not at weeks $4,8,26,39$, and 52 of treatment. The improvement rates of cephalic plaques reached $66.7 \%$ (95\% CI 51.1-80.0\%) at week 52 of treatment, with no statistically significant difference between adults and children at any weeks of treatment (Fig. 4b). Hypomelanotic macules could be assessed in 18 patients, 17 of whom were children. The improvement rate of hypomelanotic macules was $72.2 \% \quad(95 \%$ CI $46.5-90.3 \%)$ in these patients at week 52 of treatment (Fig. 4c); the relevant adult was adjudicated to "slightly improved."

Concerning the improvement rates of angiofibromas, patients who received an oral mTORC1 inhibitor (sirolimus or everolimus) concurrently with the gel were compared with those who did not. Consequently, the rate at week 52 of treatment was $76.5 \% \quad(95 \%$ CI $50.1-93.2 \%$ ) in the former in contrast to $78.6 \%$ (95\% CI 67.1-87.5\%) in the latter, thus showing no statistically significant difference at any weeks of treatment (Fig. 4d). In addition, no statistically significant difference was found at any weeks of treatment with respect to the improvement rates of angiofibromas between patients who were treated with topical petrolatum or heparinoid (Hirudoid ${ }^{\circledR}$ ) and those who were not (Fig. 7a, b).

The photographs of patients with typical skin lesions of TSC are shown in Fig. 5. A 17-year-old female (Fig. 5a) had severe angiofibromas, and her nose was totally covered by myriad reddish nodules. However, angiofibromas improved so much that the nasal contours could be identified at week 52 of treatment. A plaque on the temple resolved nearly completely and became flat at week 52 of treatment in a 22 -year-old female (Fig. 5b), although faint reddishness persisted. In a 10-year-old female (Fig. 5c), a hypomelanotic macule on the forehead disappeared completely at week 52 of treatment and became indistinct in color from the peripheral normal skin.

The Dermatology Life Quality Index (DLQI)/ Children's DLQI (CDLQI) scores changed little from the baseline scores. At baseline, 55 of 94 patients had a score 0 or could not be assessed. 


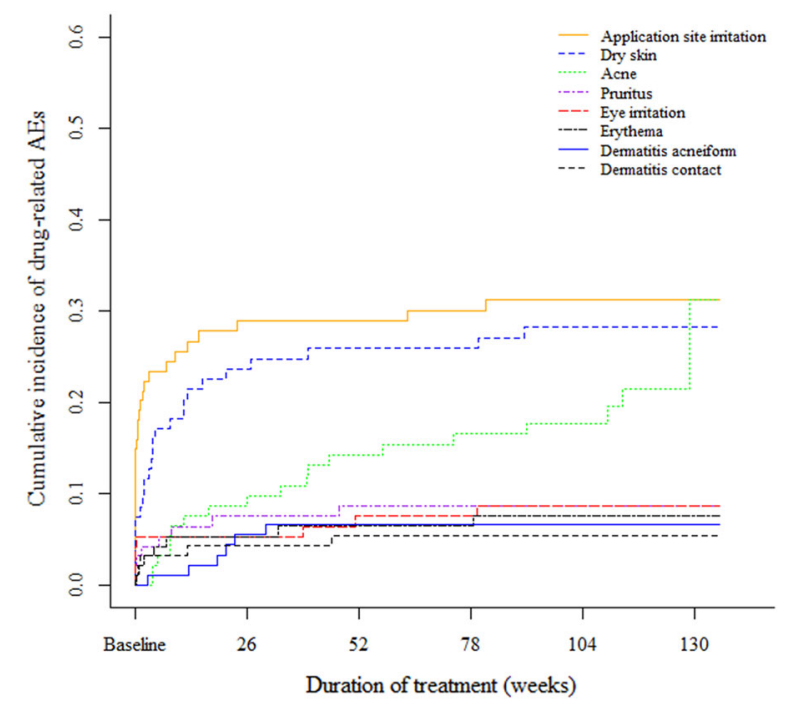

Fig. 2 Cumulative incidences of drug-related AEs. The $0.2 \%$ sirolimus gel was applied twice daily to 94 patients with TSC for a maximum of 136 weeks, and 8 drug-related AEs (incidence: $\geq 5 \%$ ) expressed with the preferred terms of MedDRA were estimated by the Kaplan-Meier method and plotted separately. Event was represented by the day when an $\mathrm{AE}$ occurred and censored by the day of discontinuing or terminating the application of the gel. TSC tuberous sclerosis complex, MedDRA the preferred terms of the Medical Dictionary for Regulatory Activities, $A E s$ adverse events

At week 52 of treatment, $89.8 \%$ of patients (95\% CI $81.5-95.2 \%$ ) provided a questionnaire response of "very satisfied" or "satisfied" in terms of patient's satisfaction.

Blood sirolimus concentrations during the trial period were $<1 \mathrm{ng} / \mathrm{ml}$ in most patients. The scattered plots of the following stratification factors were prepared: "male/female," "adults/children" (Fig. 6a, b), "yes/no" regarding the concurrent use of topical petrolatum and heparinoid (Fig. 7c, d), "present/absent" regarding drug-related AEs, and "improved/ nonimproved" regarding angiofibromas (Fig. 8a, b). Consequently, blood sirolimus concentrations did not show any statistically significant differences regarding all of these stratification factors.

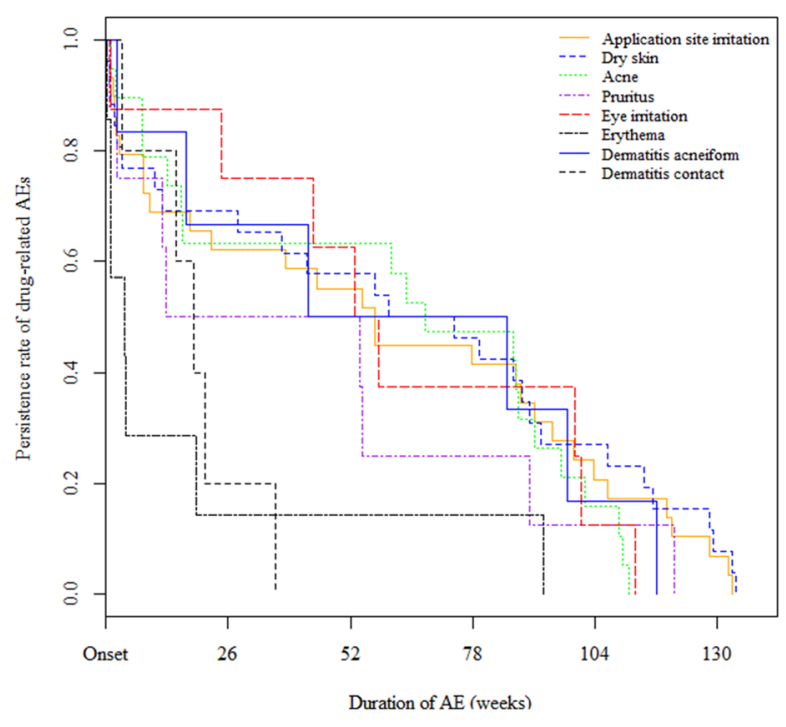

Fig. 3 Kaplan-Meier curves of the survival rates of drugrelated AEs. The sirolimus $0.2 \%$ gel was applied to 94 patients with TSC for a maximum of 136 weeks. The durations-lasting from the day of onset to the day of recovery or disappearance-of eight drug-related AEs (incidence: $\geq 5 \%$ ) expressed with the preferred terms of MedDRA were plotted separately. Event was expressed by the day when an AE recovered or disappeared, and censor by the day of discontinuing or terminating the application. AEs adverse events, TSC tuberous sclerosis complex, MedDRA Medical Dictionary for Regulatory Activities

\section{DISCUSSION}

We conducted this trial in 94 patients with TSC involving facial skin lesions to primarily examine its safety for up to 136 weeks and secondarily its efficacy for up to 52 weeks. The incidence of the primary safety endpoint was $2.1 \%$ ( 2 patients): one case of eye irritation and erythema and one case of dermatitis contact. Both AEs were mild in severity, occurred within 1 week after treatment onset, and resolved rapidly after discontinuation without requiring clinical intervention.

The most predominant drug-related AEs were application site irritation and dry skin, followed by acne. Our previous phase 3 clinical trial [15] showed the following outcomes: (1) the incidence of application site irritation was not statistically significant between the gel and placebo groups, suggesting that alcohol 
a

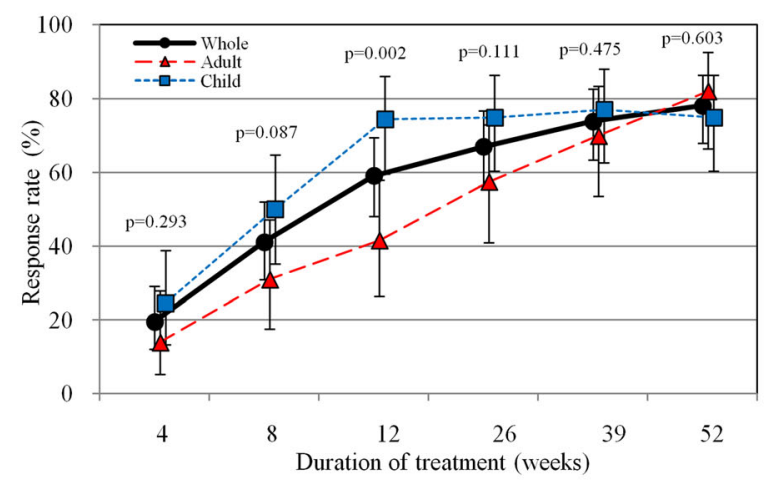

C

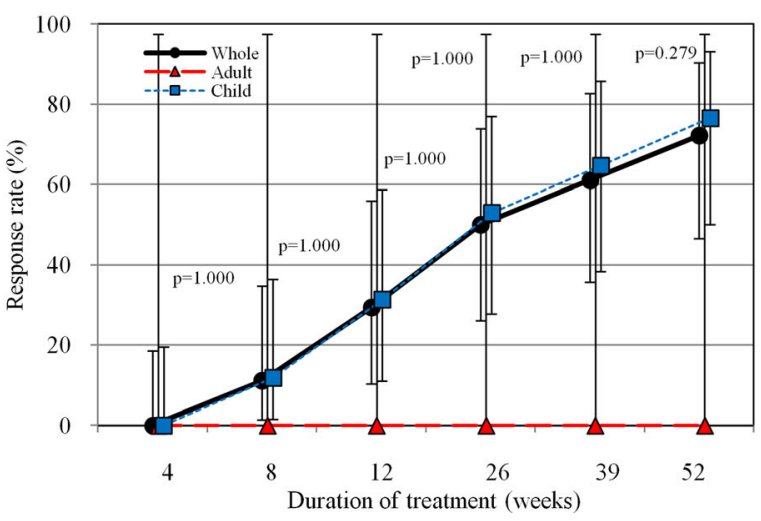

Fig. 4 Time-course changes in the response rates of skin lesions of patients with TSC. a Angiofibromas. The whole group $(n=92)$, adults $(n=43)$, and children $(n=49)$ are shown. A statistically significant difference (Fisher's exact test) was found between adults and children at week 12. b Cephalic plaques. The whole group $(n=47)$, adults $(n=16)$, and children $(n=31)$ are shown. $\mathbf{c}$ Hypomelanotic macules. The whole group $(n=18)$, one adult, and

contained in the gel base was the major cause, and (2) the incidence of dry skin was significantly greater in the gel group than in the placebo group, suggesting that sirolimus itself was the major cause thereof. Moreover, oral sirolimus was reported to have caused acne at an incidence of $>20 \%$ (Pfizer, Rapamune ${ }^{\circledR}$ Label 2018 [21]). Hence, we deem that sirolimus itself caused acne in this study as well. In most patients, any drug-related AEs were not so severe as to cause treatment discontinuation. Furthermore, drug-related AEs differed not in event type but in incidence between adults and children (data not shown). b

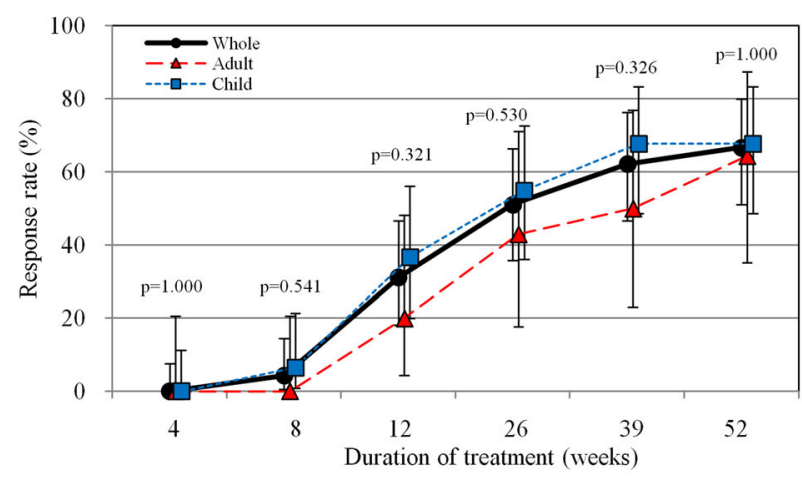

d

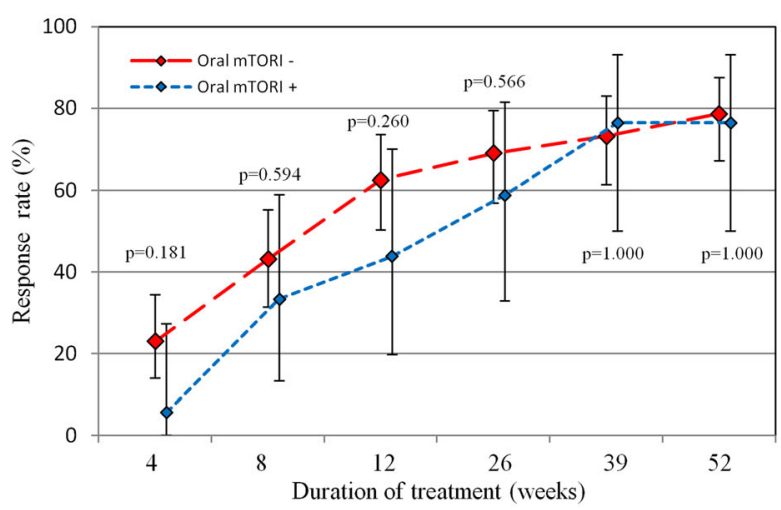

children $(n=17)$ are shown. $\mathbf{d}$ Oral mTORC1 inhibitors. The response rates of the $0.2 \%$ sirolimus gel were calculated as proportions of patients who were adjudicated by the IRC to "markedly improved" or "improved." Bars represent the $95 \%$ confidence intervals. TSC tuberous sclerosis complex, mTORC1 mammalian target of rapamycin complex $1, I R C$ independent review committee

Erythema was found in two patients, and their investigators reported the symptom as solar dermatitis and photosensitivity. However, drug-related photosensitivity was not considered definitely diagnosed based on its clinical course to be described below. In the former patient, the application of the gel was interrupted but was resumed after the complete resolution of the $\mathrm{AE}$, and the $\mathrm{AE}$ did not recur thereafter. In the latter patient, the AE persisted up to the end of the study period; however, the patient received the gel continuously without causing the further deterioration of erythema. Sirolimus was considered not to possess 
(A) Patient 1
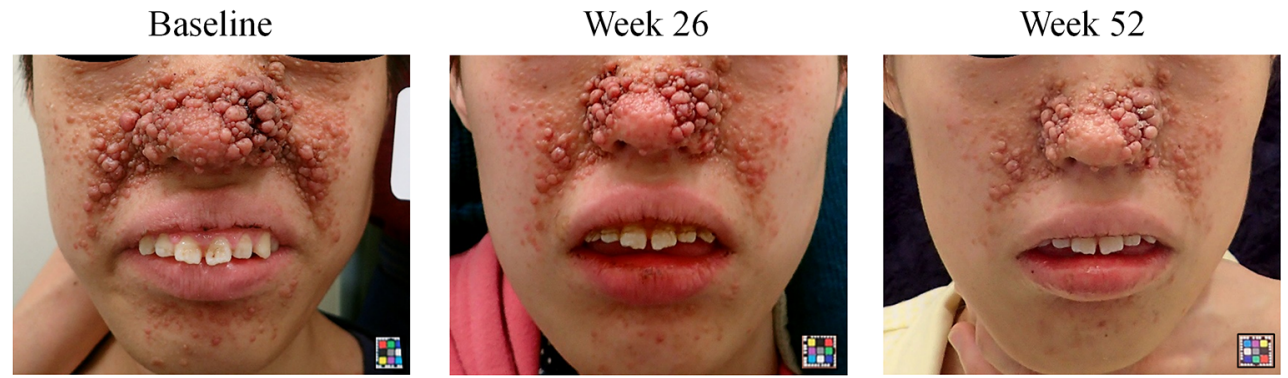

(B) Patient 2
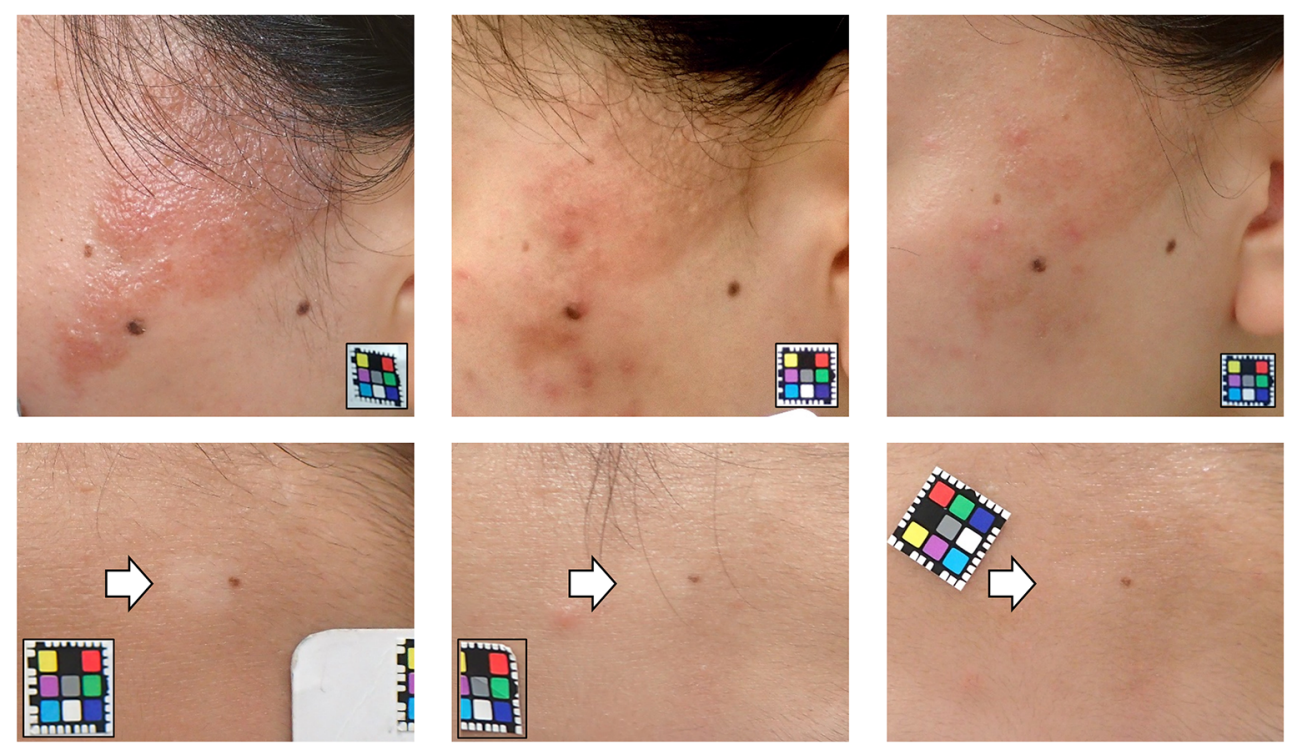

Fig. 5 Photographs of three patients with typical skin lesions of TSC. The $0.2 \%$ sirolimus gel was applied twice daily, and photographs were taken at baseline and at weeks 26 and 52 of treatment. a A 17 -year-old female had severe angiofibromas, and her nose was totally covered by myriad reddish nodules at baseline. The nodules and reddishness reduced over time at weeks 26 and 52 of treatment. b A 22 -year-old female had a relatively large plaque on the left

phototoxic potential in an in vitro study (Pharmaceuticals and Medical Devices Agency [PMDA], Rapalimus ${ }^{\circledR}$ Gel 2018) [22]. On the other hand, guinea pigs were given the $0.8 \%$ sirolimus gel used as an inducer under exposure to ultraviolet rays. Subsequently, the animals were photochallenged with the $0.05 \%$ or $0.2 \%$ sirolimus gel or vehicle. Erythema was observed at the UVA-irradiated site (PMDA, Rapalimus ${ }^{\circledR}$ Gel $2018^{19}$ ). In consideration of the abovementioned arguments, further clinical investigation will be required to definitely elucidate the potential risk of causing photosensitivity.

temple. The plaque reduced in elevation over time. At week 52 of treatment, the plaque resolved nearly completely and became flat, although faint reddishness persisted. c A 10-year-old female had a hypomelanotic macule (white arrow) on the forehead at baseline. Over time, the macule became unclear and indistinct in color from the peripheral normal skin. Patients consented to publication. TSC tuberous sclerosis complex

The improvement rates of all of angiofibromas, cephalic plaques, and hypomelanotic macules increased with time. In children, angiofibromas and cephalic plaques tended to improve in a short period of time. However, the final improvement rates of these skin lesions at week 52 of treatment did not differ between adults and children. We consider that the gel can be effective in a shorter period of time for children who have abundant blood vessels and less thickened fibrous tissue. However, we deem that the long-term application of the gel would be sufficiently effective also for adults who have 

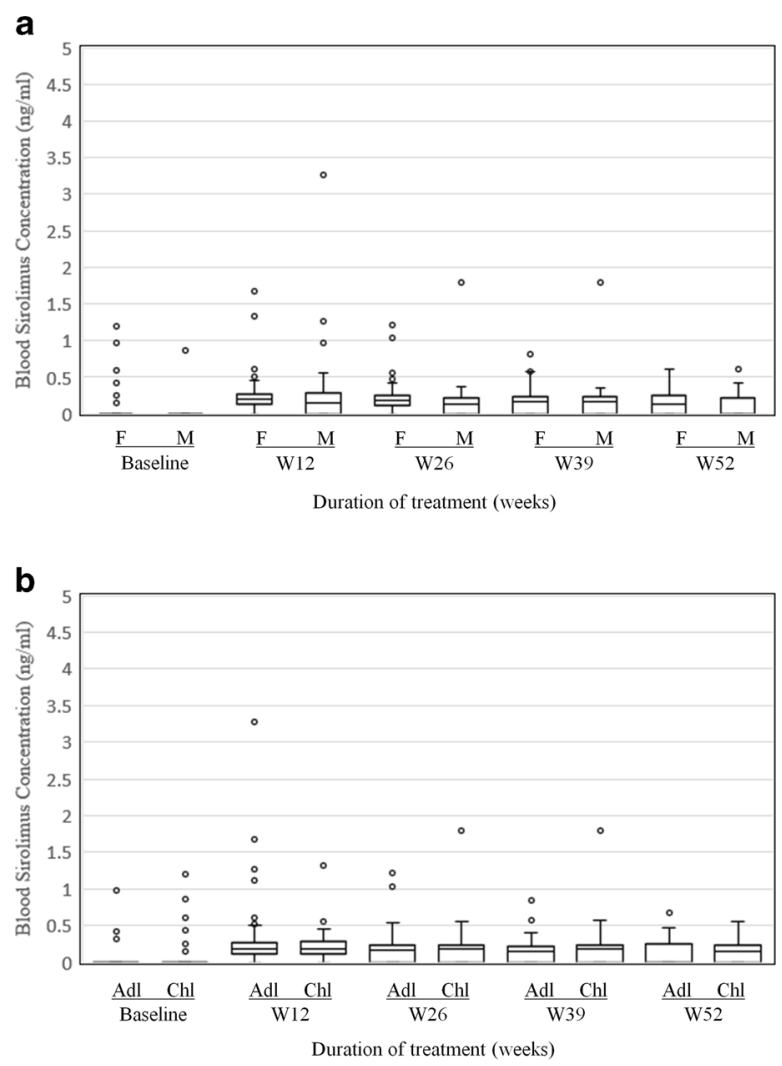

Fig. 6 Blood sirolimus concentrations in relation to sex and age. Blood sirolimus concentrations measured by LC/MS/ MS at every scheduled visit are shown in the form of boxand-whisker plots. The lower limit of detection was set to $0.1 \mathrm{ng} / \mathrm{ml}$, and a measured value of $<0.1 \mathrm{ng} / \mathrm{ml}$ is expressed as 0 . The upper and lower borders of the boxes indicate the first and third quantiles, respectively. The median is indicated as a line within the boxes, and the whiskers represent the maximum and minimum. The outliers are shown as circles outside the whiskers. a Patients are plotted for a stratification factor-sex ( $F=$ female, $M=$ male). b Patients are plotted for a stratification factor-adults (Adl, $\geq 19$ years)/children (Chl, 3-18 years). LC/MS/MS liquid chromatography/mass spectrometry

less abundant blood vessels and thickened fibrous tissue. The improvement rates of angiofibromas were analyzed according to the concurrent use or not of oral mTORC1 inhibitors. Consequently, no obvious difference was found between the two groups. Some case reports have described the effect of oral mTORC1 inhibitors on angiofibromas [23, 24]. We examined whether any additive effect in response was produced by combining oral and topical sirolimus. However, Kitayama et al. [25] reported that orally administered sirolimus in mice scarcely transferred from the blood into the skin but that topically applied sirolimus had high transdermal permeability and tissue accumulation at the application site. Response to sirolimus may be determined predominantly not by its blood concentration but its tissue concentration. This finding is in line with our experience in the clinical settings-oral sirolimus has mild effects on angiofibromas of patients with TSC who are treated with the drug alone and requires a longer administration period before exerting clinically obvious effects when compared with the case of topical application alone. Therefore, we speculate that response to topical administration might have masked that to oral administration. Hypomelanotic macules improved on a time-course basis in children. Some children including one patient in Fig. 5c exhibited dramatic improvements in hypomelanotic macules that became indistinct in color from the peripheral normal skin. The detailed mechanism by which a topical mTORC1 inhibitor acts on hypomelanotic macules remains unclear. The mTORC1 signaling was defined to control the transcription of melanogenic enzymes and melanosome maturation [26]. Histochemical examination of hypomelanotic macules of TSC patients revealed that melanocytes were present but the number of melanin granules per melanocyte decreased in TSC-related hypomelanotic macules and that sirolimus increased the volume and number of melanin granules thorough autophagy and ER stress [27-29]. The results from this study are in line with these findings.

The associations of blood sirolimus concentrations with stratification factors-sex, adults/ children, and yes/no regarding the concurrent use of topical petrolatum or heparinoid-were examined. Consequently, blood sirolimus concentrations did not show any differences regarding all of these stratification factors. In particular, topical petrolatum or heparinoid, which was used concurrently with the gel, was a moisturizer to prevent and treat skin irritation and dry skin caused by the gel. Any obvious difference was not found in the improvement 
a

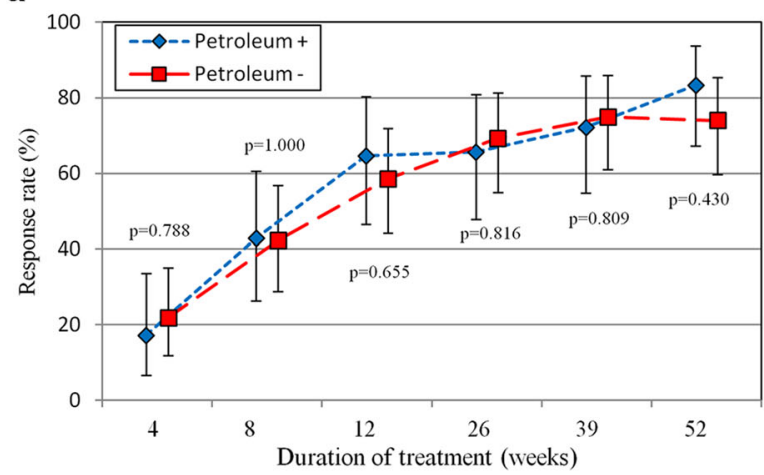

C

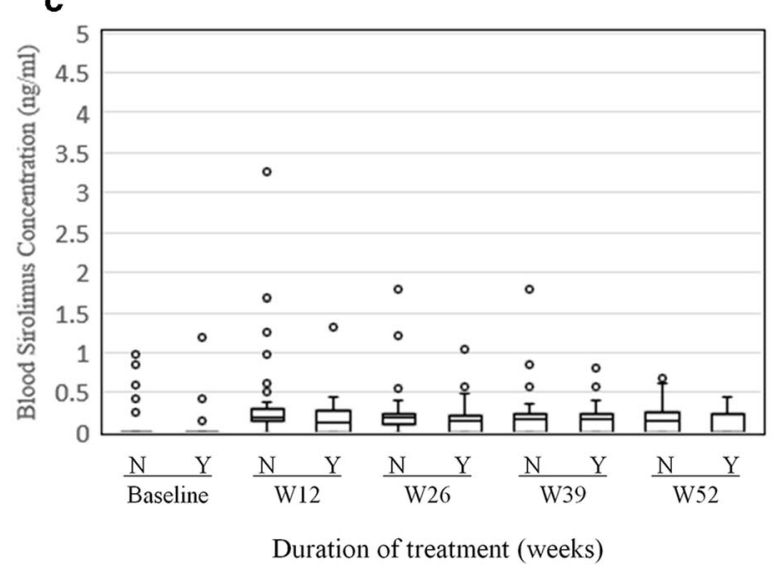

Fig. 7 Effects of the concurrent use of the $0.2 \%$ sirolimus gel and topical moisturizers. Time course changes in the response rates of angiofibromas by the concurrent use of topical petrolatum (a) or (b) heparinoid. Blood sirolimus concentrations are shown in the form of box-and-whisker plots for a stratification factor-topical petrolatum (c) or (d) heparinoid (Hirudoid ${ }^{\circledR}$ ) - which was used concurrently as a moisturizer $(\mathrm{N}=$ no, $\mathrm{Y}=$ yes $)$. Blood sirolimus concentrations were measured by LC/MS/MS at every

rates of angiofibromas (Fig. 7a, b) and blood sirolimus concentrations (Fig. 7c, d) when using or not using topical petrolatum or heparinoid, although skin sirolimus concentrations could not be measured directly. Therefore, we speculate that the use of a moisturizer affects neither the transfer of sirolimus into the skin nor its efficacy. In pediatric patients, tumor size reduced rapidly up to week 12 of application but slowly thereafter. In adult patients, on the other hand, tumor size did not reduce rapidly up to week 12 of application but continued to reduce in a nearly linear fashion. Consequently,
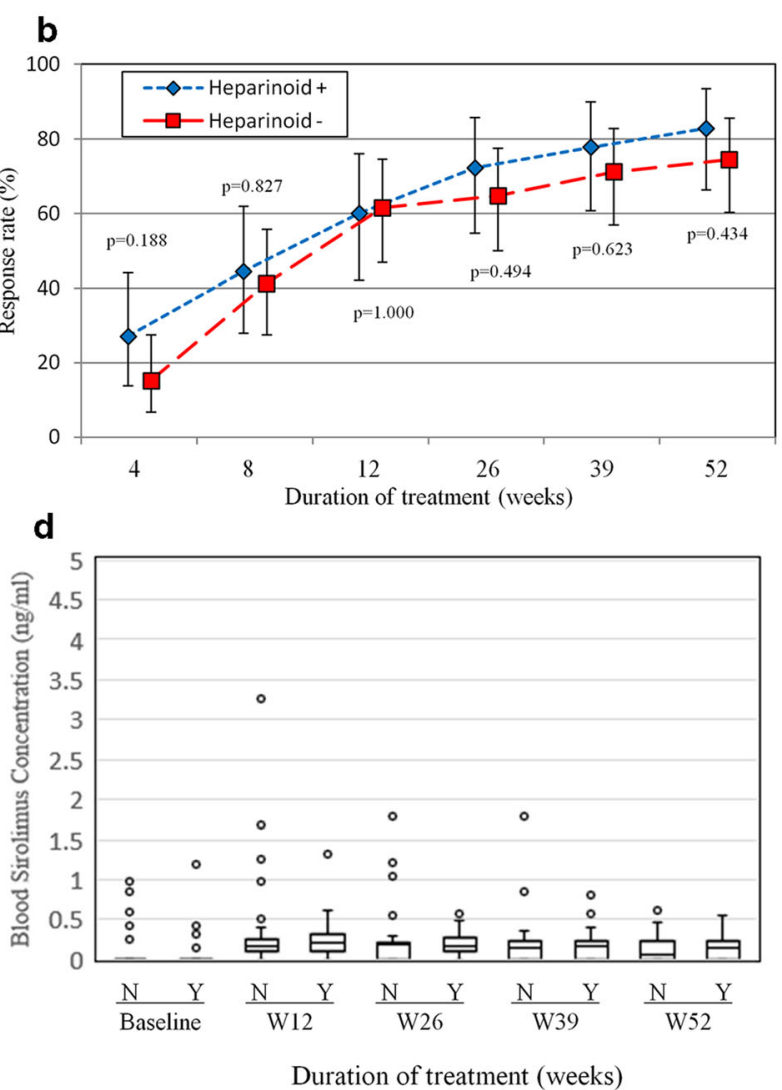

scheduled visit. The lower limit of detection is set to $0.1 \mathrm{ng} / \mathrm{ml}$, and a measured value of $<0.1 \mathrm{ng} / \mathrm{ml}$ is expressed as 0 . The upper and lower borders of the boxes indicate the first and third quantiles. The median is indicated as a line within the boxes, and the whiskers represent the maximum and minimum. The outliers are shown as circles outside the whiskers. LC/MS/MS, liquid chromatography/mass spectrometry

tumor size reduced to the comparable extent in pediatric and adult patients at week 52 of application. The response rate at week 12 of application in the present clinical study was significantly higher in children than in adults, which was similar to the result in our previous phase 3 randomized clinical trial [15]. At week 52 of application, however, no statistically significant difference was found at all in response rate between pediatric and adult patients. We consider that this difference in response pattern between them is attributable to the following facts: angiofibromas in children are reddened 

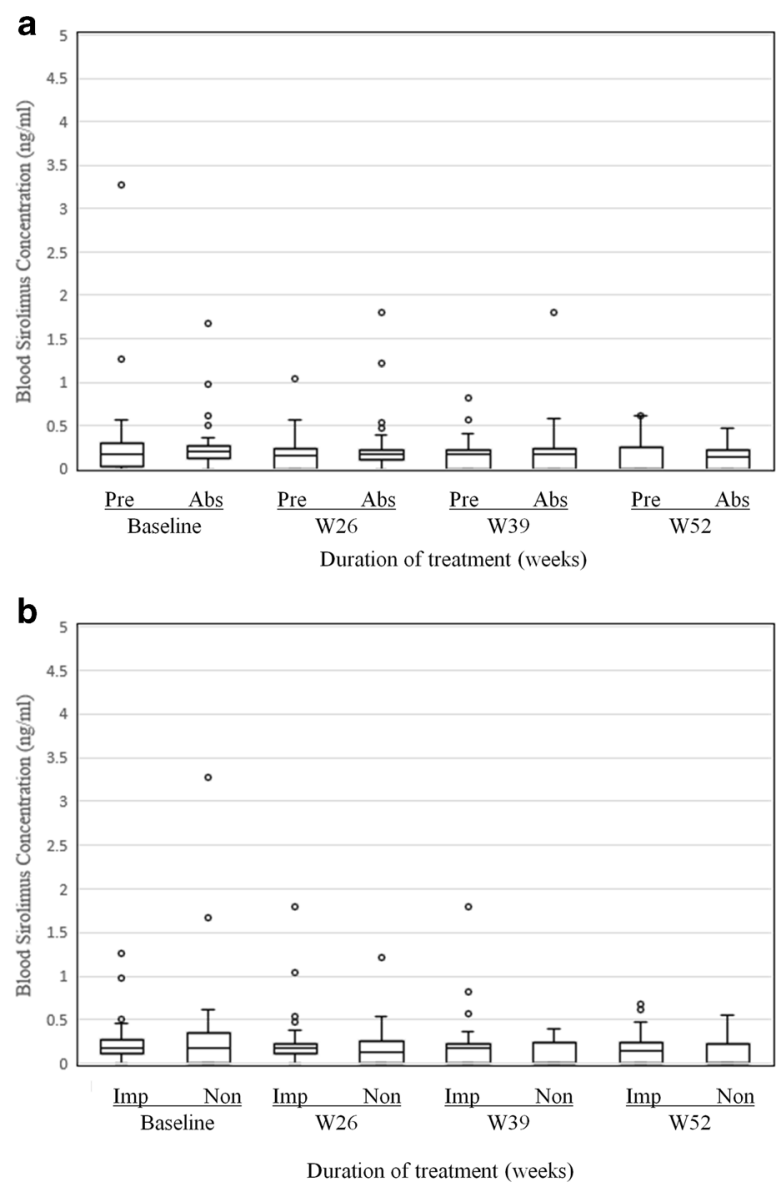

Fig. 8 Blood sirolimus concentrations in relation to drugrelated AEs and improvements in angiofibromas. Blood sirolimus concentrations measured by LC/MS/MS at every scheduled visit are shown in the form of box-and-whisker plots. The lower limit of detection was set to $0.1 \mathrm{ng} / \mathrm{ml}$, and a measured value of $<0.1 \mathrm{ng} / \mathrm{ml}$ is expressed as 0 . The upper and lower borders of the boxes indicate the first and third quantiles. The median is indicated as a line within the boxes, and the whiskers represent the maximum and minimum. The outliers are shown as circles outside the whiskers. a Patients are plotted by a stratification factor"Present (Pre)/Absent (Abs)" regarding drug-related AEs. b Patients are plotted by a stratification factor- "Improved (Imp)/Nonimproved (Non)” regarding angiofibromas that were assessed by the IRC. The "improvement" group includes patients who were adjudicated to "Markedly improved" or "Improved." AEs adverse events, $L C / M S / M S$ liquid chromatography/mass spectrometry, $I R C$ independent review committee

and soft because of abundant vasculature and less fibrous tissue components (e.g., collagen fiber). Sirolimus, when applied topically to such lesions, first and rapidly reduces tissues having abundant vessels and then slowly diminishes indurated fibrous tissues as well. On the other hand, angiofibromas in adults, which are abundant in fibroblasts and fibrous tissue components, are of skin color and frequently indurated. The drug is considered to decrease the lesions gradually. The findings from the present study suggest that the sirolimus gel, when applied for a long period of time, may be effective for sclerotized angiofibromas although a time lag can occur in the exertion of clinical effects between pediatric and adult patients with TSC. Additionally, patients who developed drug-related AEs did not necessarily show elevated blood concentrations of sirolimus (Fig. 8a). We presume that this is attributable to the following facts: (1) most drug-related AEs occurred locally, (2) blood sirolimus concentrations were below $1 \mathrm{ng} / \mathrm{ml}$ in most patients, and (3) blood sirolimus concentrations were by far lower than $5 \mathrm{ng} / \mathrm{ml}$ (Rapamune ${ }^{\circledR}$ (sirolimus) Label) - the lower limit of the effective therapeutic window to systematically administer sirolimus for the treatment of LAM. No association was found between the effects of sirolimus on angiofibromas and its blood concentrations as well (Fig. 8b). We consider that these observations provide clinical evidence that the gel exerts its therapeutic effects directly on the skin, not through the blood. At baseline, more than half of patients had the score 0 or could not be assessed with the DLQI/CDLQI questionnaires.

We consider that assay sensitivity to cutaneous lesions was low as suggested by us [15] and Koenig et al. [30]. Regarding patients' satisfaction about treatment outcomes assessed with the questionnaires, $>80 \%$ of patients were satisfied with the gel treatment at week 52 of treatment. On the other hand, a few patients were unsatisfied with the gel treatment because they had a poor feeling of usage or skin lesions did not resolve completely in 12 months of treatment.

This study has some limitations. First, the interpretation of efficacy and safety outcomes is limited because the present study had a one- 
arm, open-label and nonrandomized design. Second, the sample size was relatively small.

\section{CONCLUSIONS}

The twice-daily long-term application of the sirolimus gel was well tolerated by patients with TSC and improved angiofibromas, cephalic plaques, and hypomelanotic macules. In conclusion, we consider that the gel can be used safely for TSC patients while maintaining its efficacy when applied for a long period of time.

\section{ACKNOWLEDGEMENTS}

We are grateful to the patients, their families, and Maemi Hiraoka, the representative of the patients' association, TS Tsubasa-no-Kai, for their cooperation with the trial. The IRC members-Chikako Nishigori, MD, PhD, Division of Dermatology, Department of Internal Related, Kobe University Graduate School of Medicine, Daisuke Tsuruta, MD, PhD, Department of Dermatology, Osaka City University Graduate School of Medicine, and Nanako Yamada, MD, $\mathrm{PhD}$, Center for Clinical Residency Program, Tottori University Hospital-provided adjudication of the photographs and received honoraria from the study sponsor. Satoshi Sakima, MD, MEC Ltd., provided linguistic review of the manuscript and compensated by a study sponsor.

Funding. This study was supported and funded by Nobelpharma Co., Ltd., and in part by grants $27-Y a k u$, No. 369 orphan drug designation from the Japanese National Institutes of Biomedical Innovation, Health, and Nutrition, which holds jurisdiction by the Japan Ministry of Health, Labor, and Welfare. The journals rapid service fee was supported by Nobelpharma Co., Ltd.

Authorship. All named authors meet the International Committee of Medical Journal Editors (ICMJE) criteria for authorship for this article, take responsibility for the integrity of the work as a whole, and have given their approval for this version to be published.

Disclosures. Mari Wataya-Kaneda received grants and speaker honoraria from and has served as advisor for clinical trials sponsored by Nobelpharma, has received speaker honoraria from Novartis, and Sanofi, and Sumitomo Dainippon Pharma; Hiroshi Nagai has received grants from Nobelpharma; Yuuki Ohno has received grants from Nobelpharma; Hiroo Yokozeki has received grants from Nobelpharma; Yasuyuki Fujita has received grants from Nobelpharma; Hironori Niizeki has received grants from Nobelpharma; Kazue Yoshida has received grants from Nobelpharma and Pigeon Home Products Corporation; Masaaki Ogai has received grants from Nobelpharma; Yuichi Yoshida has received grants from Nobelpharma; Akihiko Asahina has received grants from Nobelpharma; Kazuyoshi Fukai has received grants from Nobelpharma; Chiharu Tateishi has received grants from Nobelpharma; Izumi Hamada, Tatsuro Takahata, Kenji Shimizu, and Shigeki Shimasaki are employees of Nobelpharma; Hiroyuki Murota has served as advisor in clinical trials sponsored by Nobelpharma and has received personal fees from Chugai Pharmaceutical.

Compliance with Ethics Guidelines. This study was conducted in accordance with the principles of the Declaration of Helsinki of 1964 and its later amendments and in compliance with clinical practices and local regulatory requirements. This study was approved by the institutional review board (IRB) at each institution (IRB of Osaka University Hospital, IRB of Kobe University Hospital, IRB of Tokyo Medical and Dental University Medical Hospital, IRB of JR Tokyo General Hospital, IRB of Hokkaido University Hospital, IRB of National Center for Child Health and Development, IRB of Seirei Hamamatsu General Hospital, IRB of Tottori University Hospital, IRB of the Jikei University Hospital and IRB of Osaka City University Hospital). Prior to enrollment, written informed consent was obtained from competent adult patients and from the legal representatives of patients younger than 20 years of age or 
patients with intellectual impairment. Patients also provided consent for publication.

Prior Presentation. Content within this article has been presented at the following meeting: 49th European Society for Dermatological Research Annual Meeting 18-21 September 2019 at Bordeaux, France.

Data Availability. The datasets during and/ or analyzed during the current study are available from the corresponding author on reasonable request.

Open Access. This article is licensed under a Creative Commons Attribution-NonCommercial 4.0 International License, which permits any non-commercial use, sharing, adaptation, distribution and reproduction in any medium or format, as long as you give appropriate credit to the original author(s) and the source, provide a link to the Creative Commons licence, and indicate if changes were made. The images or other third party material in this article are included in the article's Creative Commons licence, unless indicated otherwise in a credit line to the material. If material is not included in the article's Creative Commons licence and your intended use is not permitted by statutory regulation or exceeds the permitted use, you will need to obtain permission directly from the copyright holder. To view a copy of this licence, visit http://creativecommons.org/licenses/by$\mathrm{nc} / 4.0 /$.

\section{REFERENCES}

1. Krueger DA, Northrup H, International Tuberous Sclerosis Complex Consensus Group. Tuberous sclerosis complex surveillance and management: recommendations of the 2012 International Tuberous Sclerosis Complex Consensus Conference. Pediatr Neurol. 2013;49(4):255-65.

2. Northrup H, Koenig MK, Pearson DA, Au KS. Tuberous sclerosis complex. GeneReviews(R), 1999 Jul 13 [Updated 2018 July 12]. Available at https:// www.ncbi.nlm.nih.gov/books/NBK1220/. Accessed May 2, 2019.
3. Wataya-Kaneda M, Tanaka M, Hamasaki T, Katayama I. Trends in the prevalence of tuberous sclerosis complex manifestations: an epidemiological study of 166 Japanese patients. PLoS ONE. 2013;8(5):e63910.

4. Schwartz RA, Fernández G, Kotulska K, Jóźwiak S. Tuberous sclerosis complex: advances in diagnosis, genetics, and management. J Am Acad Dermatol. 2007;57(2):189-202.

5. Wataya-Kaneda M, Yano K, Hosokawa K, Yoshikawa K. A case of tuberous sclerosis with a giant angiofibroma. J Dermatol. 1997;24(2):132-4.

6. Webb DW, Clarke A, Fryer A, Osborne JP. The cutaneous features of tuberous sclerosis: a population study. Br J Dermatol. 1996;135(1):1-5.

7. Teng JM, Cowen EW, Wataya-Kaneda $\mathrm{M}$, et al. Dermatologic and dental aspects of the 2012 international tuberous sclerosis complex consensus statements. JAMA Dermatol. 2014;150(10): 1095-101.

8. Franz DN, Belousova E, Sparagana S, et al. Efficacy and safety of everolimus for subependymal giant cell astrocytomas associated with tuberous sclerosis complex (EXIST-1): a multicentre, randomised, placebo-controlled phase 3 trial. Lancet. 2013;381(9861):125-32.

9. Bissler JJ, Kingswood JC, Radzikowska E, et al. Everolimus for angiomyolipoma associated with tuberous sclerosis complex or sporadic lymphangioleiomyomatosis (EXIST-2): a multicentre, randomised, double-blind, placebo-controlled trial. Lancet. 2013;381(9869):817-24.

10. French JA, Lawson JA, Yapici Z, et al. Adjunctive everolimus therapy for treatment-resistant focalonset seizures associated with tuberous sclerosis (EXIST-3): a phase 3, randomised, double-blind, placebo-controlled study. Lancet. 2016;388(10056): 2153-63.

11. McCormack FX, Inoue Y, Moss J, et al. Efficacy and safety of sirolimus in lymphangioleiomyomatosis. N Engl J Med. 2011;364(17):1595-606.

12. DeKlotz CM, Ogram AE, Singh $S$, Dronavalli $S$, MacGregor JL. Dramatic improvement of facial angiofibromas in tuberous sclerosis with topical rapamycin: optimizing a treatment protocol. Arch Dermatol. 2011;147:1116-7.

13. Haemel AK, $\mathrm{O}^{\prime}$ Brian AL, Teng JM. Topical rapamycin: a novel approach to facial angiofibromas in tuberous sclerosis. Arch Dermatol. 2010;146(7): 715-8. 
14. Wataya-Kaneda M, Tanaka M, Nakamura A, Matsumoto S, Katayama I. A topical combination of rapamycin and tacrolimus for the treatment of angiofibroma due to tuberous sclerosis complex (TSC): a pilot study of nine Japanese patients with TSC of different disease severity. $\mathrm{Br} \mathrm{J}$ Dermatol. 2011;165(4):912-6.

15. Wataya-Kaneda M, Ohno Y, Fujita Y, et al. Sirolimus gel treatment vs placebo for facial angiofibromas in patients with tuberous sclerosis complex: a randomized clinical trial. JAMA Dermatol. 2018;154(7):781-8.

16. Tanaka M, Wataya-Kaneda M, Nakamura A, Matsumoto S, Katayama I. First left-right comparative study of topical rapamycin vs vehicle for facial angiofibromas in patients with tuberous sclerosis complex. Br J Dermatol. 2013;169(6):1314-8.

17. Wataya-Kaneda M, Nakamura A, Tanaka M, Hayashi M, Matsumoto S, Yamamoto K, Katayama I. Efficacy and safety of topical sirolimus therapy for facial angiofibromas in the tuberous sclerosis complex: a randomized clinical trial. JAMA Dermatol. 2017;153(1):39-48.

18. Northrup H, Krueger DA, International Tuberous Sclerosis Complex Consensus Group. Tuberous sclerosis complex diagnostic criteria update: recommendations of the 2012 International Tuberous Sclerosis Complex Consensus Conference. Pediatr Neurol. 2013;49(4):243-54.

19. Finlay AY, Khan GK. Dermatology Life Quality Index (DLQI) - a simple practical measure for routine clinical use. Clin Exp Dermatol. 1994;19(3): 210-6.

20. Lewis-Jones MS, Finlay AY. The children's dermatology life quality index (CDLQI): initial validation and practical use. Br J Dermatol. 1995;132(6):942-9.

21. Rapamune ${ }^{\circledR}$ (sirolimus) Label. https://labeling. pfizer.com/showlabeling.aspx?id=139. Accessed May 2, 2019.

22. Rapalimus ${ }^{\circledR}$ gel $0.2 \%$ Report on the deliberation results, PMDA 2018. https://www.pmda.go.jp/files/ 000225368.pdf. Accessed May 2, 2019.
23. Hofbauer GF, Marcollo-Pini A, Corsenca A, et al. The mTOR inhibitor rapamycin significantly improves facial angiofibroma lesions in a patient with tuberous sclerosis. Br J Dermatol. 2008;159(2): 473-5.

24. Tarasewicz A, Debska-Slizień A, Konopa J, Zdrojewski Z, Rutkowski B. Rapamycin as a therapy of choice after renal transplantation in a patient with tuberous sclerosis complex. Transplant Proc. 2009;41(9):3677-82.

25. Kitayama K, Maeda S, Nakamura A, Katayama I, Wataya-Kaneda M. Efficiency of sirolimus delivery to the skin is dependent on administration route and formulation. J Dermatol Sci. 2019;94(3):350-3.

26. Ho H, Kapadia R, Al-Tahan S, Ahmad S, Ganesan AK. WIPI1 coordinates melanogenic gene transcription and melanosome formation via TORC1 inhibition. J Biol Chem. 2011;286(14):12509-23.

27. Wataya-Kaneda M, Tanaka M, Yang L, et al. Clinical and histologic analysis of the efficacy of topical rapamycin therapy against hypomelanotic macules in tuberous sclerosis complex. JAMA Dermatol. 2015;151(7):722-30.

28. Yang F, Yang L, Wataya-Kaneda M, Yoshimura T, Tanemura A, Katayama I. Uncoupling of ER/mitochondrial oxidative stress in mTORC1 hyperactivation-associated skin hypopigmentation. J Invest Dermatol. 2018;138(3):669-78.

29. Yang F, Yang L, Wataya-Kaneda M, et al. Dysregulation of autophagy in melanocytes contributes to hypopigmented macules in tuberous sclerosis complex. J Dermatol Sci. 2018;89(2):155-64.

30. Koenig MK, Bell CS, Hebert AA, Treatment Trial Collaborators, et al. Efficacy and safety of topical rapamycin in patients with facial angiofibromas secondary to tuberous sclerosis complex: the TREATMENT randomized clinical trial. JAMA Dermatol. 2018;154(7):773-80. 